\title{
Look-up Table Reduction for Subcarrier Activation in OFDM with Index Modulation Systems
}

\author{
Abdulrahman Ikram Siddiq ${ }^{1}$, Hussein Nadhem Fadhel $^{2}$ \\ \{draisiddiq@ntu.edu.iq ${ }^{1}$, h.fadil@ntu.edu.iq ${ }^{2}$ \} \\ TECK, Northern Technical University, Kirkuk, Iraq ${ }^{1,2}$
}

\begin{abstract}
Orthogonal frequency division multiplexing with index modulation (OFDMIM) is a promising multi-carrier communication technique. A limited set of $k$ out of $n$ available subcarriers are activated and modulated by constellation symbols. The selection of the subcarrier activation pattern is performed according to data mapped via Look-up Tables (LUTs) or equivalent iterative mathematical algorithms. The complexity of mapping data to active subcarrier indices can rise dramatically with $\mathrm{n}$, and then limit the applicability of OFDM-IM. Therefore, in this paper, a new subcarrier activation scheme is proposed, where smaller size LUTs are used. A reduction ratio in LUT overall size of about $84 \%$ is achieved for $(n, k)=(8,4)$, and this ratio increases with $\mathrm{n}$. The presented analysis and simulation show that an OFDM-IM exploiting the proposed scheme is an identical less complex version of classical OFDM-IM, with a very close BER performance.
\end{abstract}

Keywords: Orthogonal frequency division multiplexing, subcarrier index modulation, subcarrier encoding, subcarrier activation pattern.

\section{Introduction}

Orthogonal Frequency Division Multiplexing (OFDM) is the multicarrier transmission a technique that has become a popular signaling format in high-speed wireless communications [1]. OFDM is adopted in many standards, such as Asymmetric Digital Subscriber Line (ADSL), Wi-Fi, Worldwide Inter-operability for Microwave Access (WiMax), Long Term Evolution (LTE) [2]. The reason is the advantages of OFDM including its high resistance to frequency selective fading, ease of implementation, high data rate, and high spectral efficiency [1,2]. OFDM with Index Modulation (OFDM-IM) systems are the result of applying the concept of spatial modulation (SM) to the subcarriers of OFDM [3]. The growing interest in OFDM-IM is due to its many advantages over OFDM. Advantages such as transmit power-saving [4-6], better BER performance [3,7], and a trade-off between system performance and spectral efficiency (SE) $[4,7]$, have made OFDM-IM a possible alternative to OFDM in future communication standards $[8,6]$.

Moreover, SM has been successfully applied in multiple antenna communication systems. That is, among a set of available transmit antennas, a unique antenna is selected to transmit constellation symbols. The selected antenna number is used as an information-bearing unit [9]. Index modulation is also used in a decision gathering scheme for cluster-based wireless sensor networks [10]. It is reported that this scheme outperforms conventional schemes in terms of overall decision error rate. In [11], a differential subcarrier index modulation scheme is proposed to eliminate the need to channel state information knowledge at the receiver. 
To increase the SE of OFDM-IM, the Multiple-Mode OFDM-IM is proposed to convey data through the use of multiple distinguishable constellations and their full permutations. The system achieves a better BER performance than OFDM-IM [12].

A modification to the data to the SAP mapping scheme is proposed in [13] such that the subcarriers are activated with an equal probability. The equiprobable subcarrier activation (ESA) involves iterative arithmetic operations and requires the small table to store SAPs in the transmitter and receiver. The complexity of ESA is comparable with the classical combinatorial scheme. The Generalized Precoding aided Quadrature Spatial Modulation (GPQSM) is proposed in [14]. It is applied on both phase and quadrature components of the signal in MIMO systems. It is shown that the improvement in BER performance of GPQSM is at the expense of an increased decoding complexity as compared with traditional MIMO. Two methods are proposed in [15] to enhance the SE and the diversity of OFDM-IM. The combinatorial data to the SAP mapping scheme is modified and applied to the in-phase and quadrature components of the signal. It is shown that the proposed methods enable low complexity detection and better error performance over classical OFDM-IM.

In more detail, the basic idea in OFDM-IM is that only a subset of the available subcarriers are selected according to the data and used to carry constellation symbols while the other subcarriers in the OFDM block are just turned off. Being selected according to data, the indices of active subcarriers also convey information [3]. To avoid high complexity, an N-subcarrier OFDM block is divided into $g$ subblocks of $n$ subcarriers in each. Subcarrier activation is applied to these subblocks independently, usually with a fixed activation ratio, $k / n$. That is $k$ of the subcarriers out of $n$ in a subblock are activated $[4,16]$. However, there are $C_{k}^{n}\left(=\frac{n !}{k !(n-k) !}\right)$ possible subcarrier activation patterns (SAPs). An SAP is selected according to $p_{1}$ bits of data, where $p_{1}=\left\lfloor\log _{2} C_{k}^{n}\right\rfloor$ and \lfloor\rfloor is the greatest lower integer operator. Then, the $k$ active subcarriers are modulated by $p_{2}=k \log _{2} M$ bits of data, where $M$ is the signal constellation order. As a result, a total of $p=\left(p_{1}+p_{2}\right)$ bits are loaded to each OFDM-IM subblock [4,15]. The $p_{1}$ to SAP mapping/demapping is implemented via Look-up Tables (LUTs) whose size becomes dramatically high for large $n[3,4,6,7]$. In [3], it is proposed to use combinatorial number theory to map data to subcarrier indices instead of LUTs for large $n$. The combinatorial algorithm selects the active subcarrier indices by calculating the binomial coefficients many times. The scheme also involves binary-to-decimal and decimal-to-binary conversion at the transmitter and receiver, respectively, therefore, the complexity is still not low. Additionally, it can give a catastrophic result in some error cases.

In this paper, a new subcarrier activation scheme is proposed where LUTs smaller in size as compared to classical OFDM-IM are used. The advantage of the proposed scheme is twofold, it reduces system complexity and avoids the need to use the combinatorial scheme. The rest of this paper is organized as follows. In Section II, the details of subcarrier encoding, decoding, and LUT sizes of the proposed subcarrier activation scheme, are described. The BER performance of an OFDM-IM exploiting the proposed scheme is evaluated in Section III. Finally, conclusions are given in Section IV.

\section{System Model}

An OFDM-IM system conveys $m(=g p)$ bits by the transmission of each $N$-subcarrier frequency domain block denoted as $\boldsymbol{x}=\left[x_{0}, x_{1}, \ldots, x_{N-1}\right]^{T}$. That is, $\boldsymbol{x}$ is the result of the subcarrier activation/deactivation and symbol modulation according to a stream of $m$ bits, as 
described in Section 1. Then, as in ordinary OFDM systems, $\boldsymbol{x}$ undergoes the processes of IFFT, Cyclic Prefix (CP) addition, transmission through the channel, CP removal and FFT, whose output is an estimate of $\boldsymbol{x}$ denoted as $\boldsymbol{y}=\left[y_{0}, y_{1}, \ldots, y_{N_{1}}\right]^{T}$. In this work, a slowly timevarying multipath Rayleigh fading channel is assumed. The equivalent frequency domain relation between $\boldsymbol{x}$ and $\boldsymbol{y}$ can be expressed as

$$
\boldsymbol{y}=\boldsymbol{H} x+\boldsymbol{w}
$$

Where $\boldsymbol{H}=\operatorname{diag}\left(h_{0}, h_{1}, \ldots, h_{N-1}\right)$ and $h_{i}$ is the channel fading coefficient at the $(i+1)^{\text {th }}$ subcarrier with $h_{i} \sim \mathcal{C} \mathcal{N}(0,1)$. The independent additive white Gaussian noise (AWGN) is $\boldsymbol{w}=$ $\left[w_{0}, w_{1}, \ldots, w_{N-1}\right]^{T}$ such that $w_{i} \sim \mathcal{C N}\left(0, N_{o}\right)$.

\section{Proposed Scheme}

The proposed scheme aims to reduce the complexity of $p_{1}$ to SAP mapping/demapping in OFDM-IM systems. The idea is to split $p_{1}$ into two segments, namely, $p_{11}$ and $p_{12}$, and map each segment to a corresponding part of the SAP in different ways.

\subsection{Subcarrier Encoding}

Subcarrier encoding is the activation/deactivation of the subcarriers of a subblock according to $p_{1}$. In the proposed scheme, the binary word $p_{1}$ is divided into two smaller size words $p_{11}$ and $p_{12}$. Such that, $p_{11}$ are the first $d$ bits of $p_{1}$, where

$$
d=\min (k, n-k)
$$

and $p_{12}$ is the remaining bits of $p_{1}$. It can be shown that $p \geq d \forall n, k$. The bits of $p_{11}$ are used to directly determine the state of the first $d$ lhs subcarriers of the subblock. Whereas, $p_{12}$ is used to select the activation pattern for the remaining $n-d r h s$ subcarriers, such that the total number of active subcarriers in the subblock is $k$.

The $2^{p_{11}}$ possible codes of $p_{11}$ can be classified into $d+1$ categories in terms of the number of $1 \mathrm{~s}$ in $p_{11}$, denoted by $n_{1}\left(p_{11}\right)$. Each category consists of $C_{i}^{d}$ codes, where, $i=n_{1}\left(p_{11}\right)$ and $0 \leq i \leq d$. The total number of codes in all of these categories is

$$
\sum_{i=0}^{d} C_{i}^{d}=2^{p_{11}}
$$

According to the proposed scheme, for each $p_{11}$ code with $i$ ones, there must be $k-i$ ones, active subcarriers, in the $r h s$ SAP. Then, for each such $p_{11}$ code, there are actually $C_{k-i}^{n-d} r h s$ SAPs. On the other hand, to achieve a total of $2^{p_{1}}$ SAPs, then for each $p_{11}$ code there must be at least $2^{p_{12}}$ different $r h s$ SAPs to be assigned to the $2^{p_{12}}$ possibilities of $p_{12}$. This necessitates that

$$
C_{k-i}^{n-d} \geq 2^{\mathrm{p}_{12}}, \quad 0 \leq \mathrm{i} \leq \mathrm{d}
$$

However, Eq.(4) is not valid for all values of $i$. That is, for some $i$ values, such as 0 and $d, C_{k-i}^{n-d}$ is less than $2^{p_{12}}$. While, fortunately, it is greater than $2^{p_{12}}$ for other $i$ values such as $d / 2$ or $(d+1) / 2$ for even and odd $d$, respectively. The residual $C_{k-i}^{n-d}-2^{p_{12}}$ SAPs can be used to 
complete the required number of $r h s$ SAPs in the cases of $C_{k-i}^{n-d}<2^{p_{12}}$. This can be correct, and hence the proposed scheme is valid for all $n$ and $k$, if the total number of generated SAPs by the proposed scheme is equal or greater than the total number of SAPs required in the system, $2^{p_{1}}$. Generally, in the proposed scheme, there are $C_{k-i}^{n-d} r h s$ SAPs for each $p_{11}$ code of $i$ ones, i. e., $C_{i}^{d} C_{k-i}^{n-d} r h s$ SAPs for each category of $p_{11}$. Then, summing over the $d+1$ categories, yields $C_{k}^{n}$, which is by definition greater than $2^{p_{1}}$.

$$
\sum_{i=0}^{d} C_{i}^{d} C_{k-i}^{n-d}=C_{k}^{n}
$$

Next, the $p_{12}$ to $r h s$ SAP mapping is performed by $d+1$ LUTs each of size $2^{\left(p_{12}\right)}(n-d)$ bits. That is, for a given $p_{11}$ with $n_{1}\left(p_{11}\right)$ ones, a LUT is used to mapping the associated $p_{12}$ to $(n-d)$ - element $r h s$ SAPs of $k-n_{1}\left(p_{11}\right)$ ones. A total of $Q_{\text {prop }}$ bits of memory is required to store the LUTs.

$$
Q_{\text {prop }}=2^{\left(p_{12}\right)}(n-d)(d+1)
$$

Whereas, in conventional OFDM-IM $p_{1}$ is mapped to n- bit SAPs by a $Q_{c o n v}$ - bit LUT.

$$
Q_{\text {conv }}=2^{p 1} \times n
$$

Bearing in mind that $p_{11}$ is directly mapped to $l$ s SAPs, Table 1 shows the superiority, defined by the percentage $\left.r=\left(Q_{\text {conv }}-Q_{\text {prop }} / Q_{\text {conv }}\right)\right) \times 100 \%$, of the proposed subcarrier activation scheme over conventional OFDM-IM. In Table $1, k=n / 2$ which produces the largest $C_{k}^{n}$ and $p_{1}$ representing the worst-case LUT size for a given $n$. The percentage advantage is about $84 \%$ at $n=8$, and $k=4$, and most importantly, it becomes greater at larger $n$. However, smaller size LUTs lead to less memory consumption, faster response, and less system complexity.

Table 1. LUT size comparison

\begin{tabular}{|c|c|c|c|c|c|c|c|c|}
\hline $\mathrm{n}$ & 2 & 4 & 6 & 8 & 10 & 12 & 16 & 32 \\
\hline$Q_{\text {conv }}$ & 4 & 16 & 96 & 512 & 1280 & 6144 & 131072 & $1.7 \mathrm{E}+10$ \\
\hline$Q_{\text {prop }}$ & 2 & 6 & 24 & 80 & 120 & 336 & 2304 & 2228224 \\
\hline$r \%$ & 50 & 62 & 75 & 84 & 90 & 94 & 98.24 & 99.99 \\
\hline
\end{tabular}

As an example, consider the case of $\mathrm{n}=8$ and $\mathrm{k}=4$. Then, $\mathrm{C}_{4}^{8}=70, \mathrm{p}_{1}=6$ bits, $\mathrm{p}_{11}=4$ bits and $\mathrm{p}_{12}=2$ bits. According to the number of ones, $0 \leq \mathrm{n}_{1}\left(\mathrm{p}_{11}\right) \leq 4$, there are 5 categories of $\mathrm{p}_{11}$ codes, with $\mathrm{C}_{\mathrm{n}_{1}\left(\mathrm{p}_{11}\right)}^{\mathrm{d}}=\mathrm{C}_{\mathrm{n}_{1}\left(\mathrm{p}_{11}\right)}^{4}=1,4,6,4$ and $1, \mathrm{p}_{11}$ codes in each category. The number of available rhs SAPs for each $p_{11}$ code depends on its $n_{1}\left(p_{11}\right)$ and is given by $C_{k-n_{1}\left(p_{11}\right)}^{n-d}=$ $\mathrm{C}_{4-\mathrm{n}_{1}\left(\mathrm{p}_{11}\right)}^{4}=1,4,6,4$ and 1 , for the five categories, respectively. There must be $2^{\mathrm{p}_{12}}=4$ rhs SAPs for each possible $\mathrm{p}_{11}$, and this is not fulfilled for the first and fifth categories. But for the third category, there are rhs SAPs more than the required number, and can be used by the first and fifth categories as long as the total number of SAPs, $\sum_{\mathrm{i}=0}^{4} \mathrm{C}_{\mathrm{i}}^{4} \mathrm{C}_{4-\mathrm{i}}^{4}=70$, is greater than $2^{\mathrm{p}_{1}}=$ 64. That is, in the third category, there are $C_{k-2}^{n-d}-2^{p_{12}}=2$ unused rhs SAPs for each possible $\mathrm{p}_{11}$ code in this category, which are $\mathrm{C}_{2}^{4}=6$ possible codes. Then, there are 12 unused SAPs in this category that can be used to complete the set of rhs SAPs in the first and fifth categories. 
The $\mathrm{p}_{12}$ to rhs mapping LUTs can be designed as follows. There are five LUTs, a LUT for each $p_{11}$ category. The LUTs of the second, third and fourth categories are as given in Table 2 .

Table 2. $p_{12}$ to $r h s$ SAP mapping of the second, third and fourth LUTs.

\begin{tabular}{|c|c|c|c|}
\hline$p_{12}$ & $r h s$ SAP 2nd LUT & $r h s$ SAP 3rd LUT & $r h s$ SAP 4th LUT \\
\hline 00 & 1110 & 0011 & 0001 \\
\hline 01 & 1101 & 0110 & 0010 \\
\hline 10 & 1011 & 1100 & 0100 \\
\hline 11 & 0111 & 1001 & 1000 \\
\hline
\end{tabular}

Note that, the patterns 1010 and 0101 are possible $r h s$ SAPs for the $n_{1}\left(p_{11}\right)=2$, not needed in the third LUT and are reserved to be used with the first and fifth LUTs of $n_{1}\left(p_{11}\right)=0$ and 4 , as shown in Table 3.

Table 3. $p_{12}$ to $r h s$ SAP mapping of the first and fifth LUTs.

\begin{tabular}{|c|c|c|c|c|}
\hline \multirow{2}{*}{$p_{12}$} & \multicolumn{2}{|c|}{1 stLUT } & \multicolumn{2}{c|}{ 5thLUT } \\
\cline { 2 - 5 } & lhs SAP & rhs SAP & lhs SAP & rhs SAP \\
\hline 00 & 0000 & 1111 & 1111 & 0000 \\
\hline 01 & 0011 & 1010 & 0011 & 0101 \\
\hline 10 & 0110 & 1010 & 0110 & 0101 \\
\hline 11 & 1100 & 1010 & 1100 & 0101 \\
\hline
\end{tabular}

The first LUT is used with a single $p_{11}$ possibility of 0000 . Then, accordingly, the only possible $r h s \mathrm{SAP}$ is 1111 . This is used when $p_{12}=00$. For the other three $p_{12}$ possibilities, the $r h s \mathrm{SAP}$ 1010 is used and to distinguish between these latter cases and the $r h s$ SAPs of $n_{1}\left(p_{11}\right)=2$, the lhs SAP is replaced by three different patterns of two ones each, to conserve a total of $k=4$ ones in the SAP. Note that these modified lhs SAPs will not interfere with the cases of $n_{1}\left(p_{11}\right)=2$ since the latter does not use the 1010 nor $0101 \mathrm{rhs}$ SAPs. Similarly, the unused $\mathrm{r}$ $\mathrm{h}$ s SAP 0101 can be used to complete the fifth LUT. That is, the fifth LUT is used when $p_{11}$ is 1111. Then, for $p_{12}=00$, an all zeros $(0000) r h s$ SAP is used. For the other three $p_{12}$ possibilities, the $r h s$ SAP is selected as 0101, with the lhs SAP cannot be left as 1111 . To have a total of $k(=4$ in this example) ones, then the lhs SAP must be replaced by three different 4 bit codes with 2 ones in each, as shown in Table 3. Such that the decoder can rely on this $l$ hs SAP to determine $p_{12}$.

\subsection{Decoding}

The subcarrier decoder demands the received SAP to estimate replicas of $p_{11}$ and $p_{12}$, denoted as $p_{11}^{\prime}$ and $p_{12}^{\prime}$, respectively. In low complexity detectors, the received SAP is detected by determining the state of each subcarrier. A better detector than simple thresholding [6] is when the $k$ highest energy subcarriers out of $\mathrm{n}$ within a received subblock are recognized as active. This scheme ensures that the detected SAP is one of the $C_{k}^{n}$ SAPs with $k$ active subcarriers and discards the $2^{n}-C_{k}^{n}$ invalid possibilities.

For simplicity, let a detected SAP be represented by an n- bit binary word, denoted by SAP'. The proposed subcarrier decoding scheme is as follows. The segment of $n-d r h s$ bits of SAP' is compared with the reserved $r h s$ patterns that are used in the LUTs of $C_{k-n_{1}\left(p_{11}\right)}^{n-d}<2^{p_{12}}$. If a 
match is found, then the corresponding LUT is selected to be used to estimate $p_{12}^{\prime}$ depending on the lhs $d$ bits of SAP', and $p_{11}^{\prime}$ is the $p_{11}$ code associated with this

LUT. Otherwise, the LUT to be used is that of $n_{1}\left(p_{11}\right)=n_{1}\left(p_{11}^{\prime}\right)$, where $p_{11}^{\prime}$ is the segment of the lhs $d$ bits of SAP'. Then, the $n-d$ pattern of SAP' is searched for in this LUT to determine $p_{12}^{\prime}$

For the example of $n=8$ and $k=4$, let SAP' $=11011000$. The rhs SAP' does not match any of the reserved patterns (1010 and 0101). Then, $p_{11}^{\prime}$ is the lhs $\operatorname{SAP}^{\prime}(=1101)$ and $n_{1}\left(p_{11}^{\prime}\right)(=3)$ selects the fourth LUT, from which the rhs SAP' is demapped to $p_{12}^{\prime}=11$. Another example is when SAP' $=00111010$. The $r h s$ SAP' $^{\prime}$ matches the reserved pattern 1010. Then, the first LUT is selected resulting in $p_{11}^{\prime}=0000$, and the $l h s \mathrm{SAP}^{\prime}$ is demapped to $p_{12}^{\prime}=$ 01.

However, there are $C_{k}^{n}-2^{p_{1}}$ unused SAPs according to the classical OFDM-IM [4,11]. In the proposed method these unused SAPs are those whose $\mathrm{r} h \mathrm{~s}$ SAP matches a reserved rhs pattern while their lhs SAPs do not match any of the cases in the corresponding LUT. For $n=8$ and $k=4$, there are six unused SAPs. Their $r h s$ SAP is either 1010 or 0101 , and their lhs SAP is one of the patterns 1001,1010 or 0101 . If such an SAP appears in the detection, due to wrong subcarrier state detection, the decoder will just consider it as an invalid SAP and discards it just like what happens in classical OFDM-IM. That is, for SAP ${ }^{\prime}=10011010$, the $r h s$ SAP matches the reserved pattern 1010. Then, the first LUT is selected and $p_{11}^{\prime}=0000$. But, the demapping of the lhs SAP' from this LUT fails, resulting in the rejection of the whole SAP'.

\section{BER Performance}

The BER performance of an OFDM-IM system exploiting the proposed subcarrier encoding scheme is evaluated by computer simulation. The k maximum energy (ME) subcarrier state detection method is used, where the $k$ highest energy subcarriers are considered as active within the received subblock. ME is adopted due to its simplicity and it ensures that a received SAP always belongs to the space of $C_{k}^{n}$ patterns of k active subcarriers. 


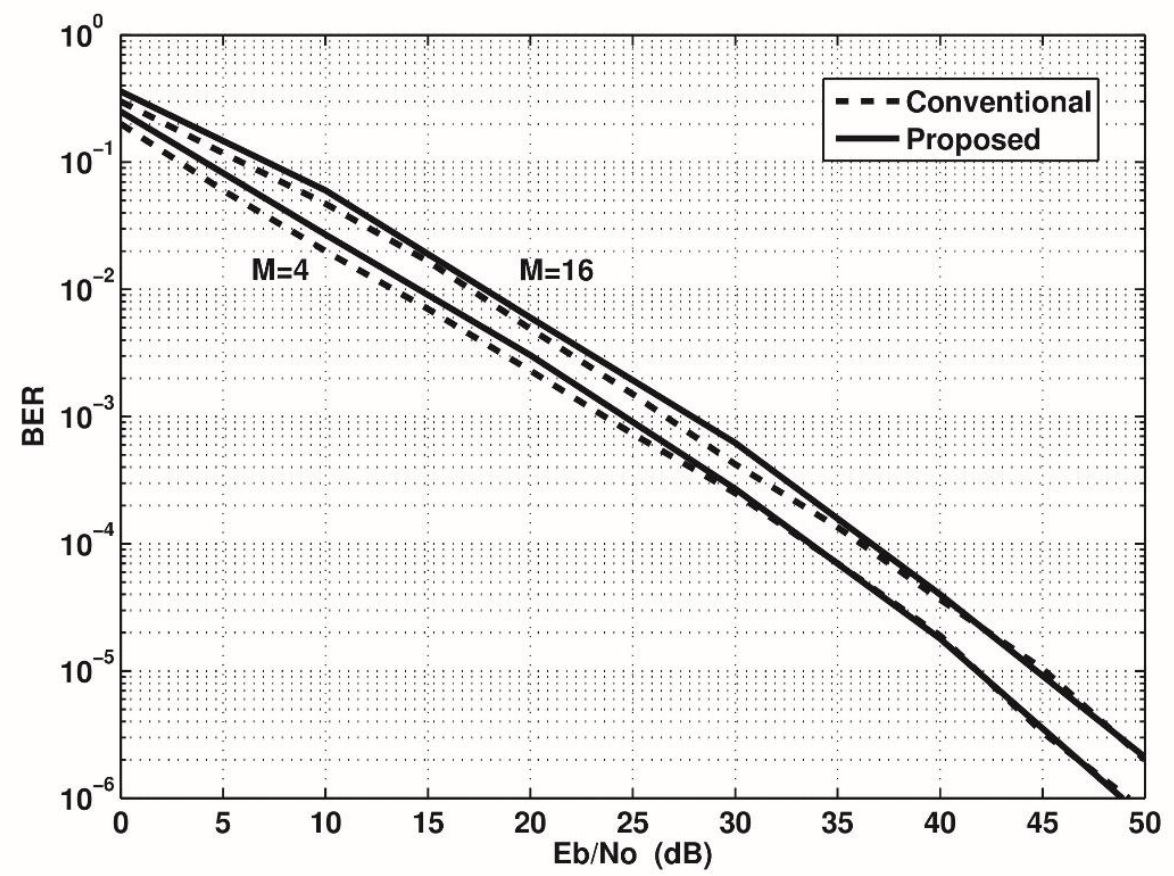

Fig 1. BER performance of OFDM-IM exploiting conventional LUT and proposed subcarrier encoding

Generally, errors in the OFDM-IM receiver result from erroneous SAP detection and demodulation of modulated active subcarriers. For proposed and classical OFDM-IM systems equivalent in terms of $n, k$, and SAP detection, a slight difference in subcarrier decoding error performance can happen due to SAP detection errors. This difference is because of the dependence in the demapping of both sides of SAP' in the proposed scheme, unlike in classical OFDM-IM. But at high enough SNR, when the state of the received subcarriers is correctly estimated, this difference should decrease and the BER performance of both systems becomes almost identical. However, active subcarrier demodulation is the same in these systems for a given SAP'. The BER is shown in Figs. 1 and 2 for $(n, k)$ of $(8,4)$ and $(10,5)$, respectively, both with $N=128, M=4$ and 16 , over a frequency selective fading Rayleigh channel. Bearing in mind that the OFDM-IM based on the proposed subcarrier encoding scheme is identical to classical OFDM-IM in terms of spectral and energy efficiency, then it can be considered as a lower complexity alternative with a closely equivalent BER performance. 


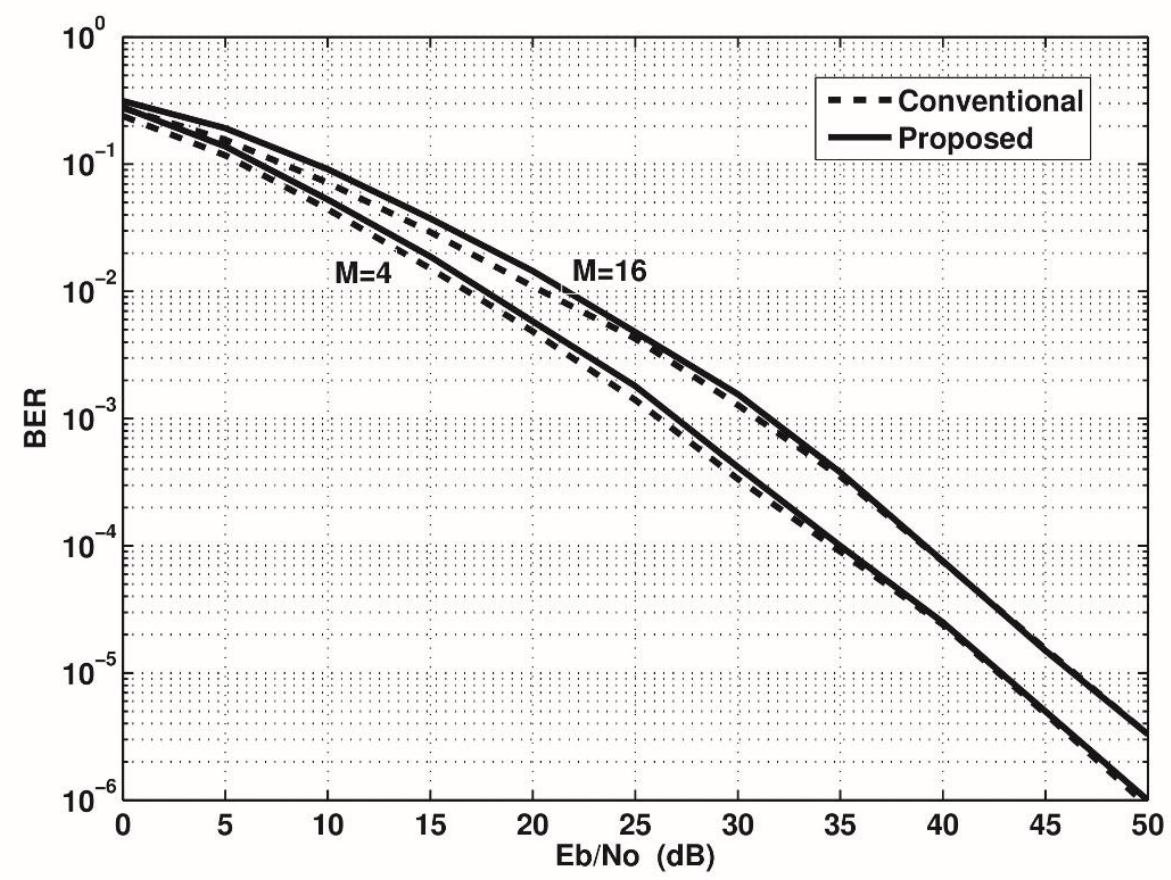

Fig 2. BER performance of OFDM-IM exploiting conventional LUT and proposed subcarrier encoding.

\section{Conclusion}

In this paper, a new subcarrier encoding scheme is proposed for OFDM-IM systems. Unlike classical OFDM-IM where a code of $p_{1}$ data bits are mapped via a LUT to an SAP, or by equivalent relatively complicated mathematical operations, it is proposed to use part of $p_{1}$ to directly activate/deactivate part of the subblock. The remaining bits of $p_{1}$ are mapped by LUTs to SAPs for the remaining part of the subblock. That is, a shorter data code is mapped and hence smaller LUTs are used concerning the LUT of an equivalent classical OFDM-IM. In the proposed scheme, the data-to-SAP mapper/demapper does not need to run an exhaustive search to the contents of a huge LUT to locate a match, but instead, one of the $(d+1)$ smaller size LUTs need to be searched. Moreover, the 1's complement symmetry between pairs of the $(d+$ 1) LUTs could be invested to implement hardware realizations with further reduction in the complexity of the proposed scheme. However, such realizations are feasible as long as their hardware implementation is more efficient as compared with the combinatorial algorithm of the same $\mathrm{n}$ and $\mathrm{k}$. This comparison depends on hardware implementation parameters which are beyond the scope of this paper. Simulation tests show the close equivalence of the proposed and conventional OFDM-IM in terms of BER performance. The proposed method can be used in OFDM-IM and its derivatives as a lower storage memory demanding alternative to the conventional LUT scheme of index bits to SAP mapping. 


\section{References}

[1] Ahmad, J., Jehad, H., Huseyin, A., 2020. OFDM With Hybrid Number and Index Modulation. IEEE ACCESS. 8, 55042-55053.

[2] Liu, H., Cong, W., Wang, L., Li, D., 2017. Symbol error rate performance of nonlinear OFDM receiver with peak value threshold over frequency selective fading channel. AEU-Int J Electron Commun. 74, 163-170.

[3] Basar, E., Aygolu, U., Panayirci, E., Poor, H., 2013. Orthogonal frequency division multiplexing with index modulation. IEEE Trans Signal Process. 61 (22), 5536-5549.

[4] Basar, E., Wen, M., Mesleh, R., Renzo, M., Xiao, Y., Haas, H., 2017. Index modulation techniques for next-generation wireless networks. IEEE Access. 5. 16693-16746.

[5] Siddiq, A., 2017. E_ect of subcarrier activation ratio on the performance of OFDM-IM over rayleigh fading channel. IEEE Commun Lett. 21 (6), 1293-1296.

[6] Mrkic, J., Kocan, E., Pejanovic-Djurisic, M., 2017. Index modulation techniques in OFDM relay systems for $5 \mathrm{G}$ wireless networks. IEEE 40th International Conference on Telecommunications and Signal Processing (TSP), 208-211.

[7] Fan, R., Yu, Y., Guan, Y., 2015. Generalization of orthogonal frequency division multiplexing with index modulation. IEEE Trans Wireless Commun, 14 (10), 5350-5359.

[8] Basar, E., 2016. Index modulation techniques for 5G wireless networks. IEEE Commun Mag. 54 (7), 168-175.

[9] Mesleh, R., Haas, H., Sinanovic, S., Ahn, C., Yun, S., 2008. Spatial modulation. IEEE Trans Veh Technol. 57 (4), 2228-2241.

[10] Althunibat, S., Mesleh, R., 2008. Index modulation for cluster-based wireless sensor networks. IEEE Trans Veh Technol. 67 (8), 6943-6950.

[11] Althunibat, S., Mesleh, R., Basar, E., 2008. Differential subcarrier index modulation. IEEE Trans Veh Technol. 67 (8), 7429-7436.

[12] Li, Q., Wen, M., Basar, E., Poor, H., Zheng, B., Chen, F., 2018. Diversity enhancing multiple-mode OFDM with index modulation. IEEE Trans Commun. 66 (8),3653-3666.

[13] Wen, M., Zhang, Y., Li, J., Basar, E., Chen, F., 2016. Equiprobable subcarrier activation method for OFDM with index modulation. IEEE Commun Lett. 20 (12), 2386-2389.

[14] Li, J., Wen, M., Cheng, X., Yan, Y., Song, S., Lee, M., 2017. Generalized precoding aided quadrature spatial modulation. IEEE Trans Veh Technol. 66 (2), 1881-1886.

[15] Wen, M., Ye, B., Basar, E., Li, Q., Ji, F., 2017. Enhanced orthogonal frequency division multiplexing with index modulation. IEEE Trans Wireless Commun. 16 (7), 4786-4801.

[16] Siddiq, A., 2016. Low complexity OFDM-IM detector by encoding all possible subcarrier activation patterns. IEEE Commun Lett. 20 (3), 446-449. 